Check for updates

Cite this: RSC Adv., 2019, 9, 37384

Received 30th September 2019 Accepted 4th November 2019

DOI: $10.1039 / \mathrm{c} 9 \mathrm{ra0} 9948 \mathrm{c}$

rsc.li/rsc-advances

\section{Sensitive detection of ascorbic acid using screen- printed electrodes modified by electroactive melanin-like nanoparticles}

\begin{abstract}
Wicem Argoubi, ${ }^{a}$ Amal Rabti, (D) ${ }^{a}$ Sami Ben Aoun (D) ${ }^{* b}$ and Noureddine Raouafi (D) *a
In this work, we report on the design of an enzyme-less sensitive and selective electrochemical electrode for ascorbic acid (AA) detection using a modified screen-printed electrode of melanin-like nanoparticles (Mel-NPs). Cyclic voltammetry shows that the melanin-modified electrode exhibits high electrocatalytic activity for ascorbic acid. The melanin-like nanoparticles serve as a shuttle to transport electrons from ascorbic acid to the electrode surface. The modified electrode is endowed with a large dynamic window ranging from 5 to $500 \mathrm{ppb}$. The detection and quantification limits were estimated to be 0.07 and 0.23 $\mathrm{ppb}$, respectively. The modified electrode was successfully used to determine AA in human blood serum, urine and saliva with satisfactory recovery levels.
\end{abstract}

\section{Introduction}

Ascorbic acid, vitamin C, is a widely used antioxidant in food, animal feed, beverages, and pharmaceutical formulations. ${ }^{\mathbf{1 , 2}}$ It takes part in many important human life processes as it is necessary for promoting the generation of antibodies and the absorption of iron. ${ }^{3}$ Therefore, developing accurate, reliable, rapid and easy to implement methods for measuring low levels of ascorbic acid in real samples is of great importance. Despite the fact that vitamin $\mathrm{C}$ is unstable in aqueous solution since it is easily oxidized reversibly to dehydroascorbic acid and subsequently irreversibly to 2,3-diketo-L-gulonic acid, ${ }^{4}$ which make it difficult to be quantified, many methods have been developed for its determination. In addition to titrimetry, ${ }^{5}$ fluorimetry, ${ }^{6,7}$ flow injection analysis, ${ }^{\mathbf{8}, 9}$ spectrophotometry, ${ }^{\mathbf{1 0 , 1 1}}$ solid-phase iodine method, ${ }^{12}$ and chromatography, ${ }^{\mathbf{1 3}, 14}$ electrochemical methods have attracted much interest because they are less sensitive towards the matrix effects than other analytical techniques and there is no need for derivatization of the analytes. ${ }^{15}$

Although ascorbic acid is electroactive, its direct detection using conventional electrodes such as glassy carbon, graphite and platinum is challenging because of its high overpotential resulting in electrode fouling, and poor selectivity and reproducibility. ${ }^{\mathbf{1 6}}$ To overcome these challenges, increased investigations have been carried out with nanomaterials modified electrodes for AA sensing. Typical examples included carbonsupported $\mathrm{NiCoO}_{2} \quad$ nanoparticles, ${ }^{17}$ branch-trunk $\mathrm{Ag}$

${ }^{a}$ Tunis El Manar University, Chemistry Department, Laboratory of Analytical Chemistry and Electrochemistry (LR99ES15), Campus Universitaire de Tunis El Manar 2092, Tunis, Tunisia. E-mail: noureddine.raouafi@fst.utm.tn

${ }^{b}$ Taibah University, Faculty of Science, Chemistry Department, P. O. Box 30002, AlMadinah Al-Munawarah, Saudi Arabia.E-mail: sbenaoun@taibahu.edu.sa hierarchical nanostructure, ${ }^{18} \mathrm{NiFe}_{2} \mathrm{O}_{4}$ nanoparticles, ${ }^{19} \mathrm{MoS}_{2}$ nanosheets, ${ }^{20} \mathrm{CuS} /$ Prussian blue core-shell nanohybrid, ${ }^{21}{ }^{4-}$ aminobenzoic acid functionalized herringbone carbon nanotubes, ${ }^{22}$ etc. Despite the enhanced performances of AA electrochemical sensors when using nanomaterials, they have not yet been successfully validated into marketed products which make it of paramount importance to construct portable, fast and highly sensitive real-time system for accurate point-of-care AA detection.

Great interests have been recently shown to melanin-like nanoparticles (Mel-NPs) because of their low-cost of production, low toxicity, high dispersibility, stability and biodegradability. ${ }^{23}$ More recently, they have been used to modify electrode to build an electrochemical sensor for a simultaneous detection of ascorbic acid, dopamine (DA) and uric acid (UA) using facile gold nanoplates and reduced graphene oxide modified glassy carbon electrode. ${ }^{24}$ The redox activity of melanin has been linked to pro-/antioxidant behavior, which makes it an excellent material for biomedical purposes. For instance, certain 'black bacteria' are protected against phagocytic mechanisms by a layer of melanin within their cell walls. ${ }^{25}$ Moreover, melanins' redox features in the solid state exhibit a fast charge time at the proper static dielectric constant which make it exploitable in electrical energy storage devices. ${ }^{26}$ Melanins have been also proposed for optoelectronic and photovoltaic applications, ${ }^{27}$ which make them interesting material with a wide range of possible technological applications.

In this work, we designed a sensitive and selective electrochemical ascorbic acid sensor using a modified screen-printed electrode by melanin-like nanoparticles, which were synthesized through neutralization of dopamine hydrochloride salt with $\mathrm{NaOH}$ followed by spontaneous air oxidation of dopamine at $50{ }^{\circ} \mathrm{C}$. Screen-printed electrodes, enabling measurements in a minimum sample volume, were chosen to allow the future 
application of the developed electrochemical sensor in the elaboration of portable point-of-care testing system with a sample-in answer-out approach that enables their use by nonspecialized personnel. Voltamperometric investigations showed that the melanin-modified electrode exhibits a high electrocatalytic activity for ascorbic acid. The voltammetric curves demonstrated excellent analytical performances with high selectivity towards ascorbic acid. The method for generating nanoparticles and electrode modification are depicted in Fig. 1.

\section{Methods}

\subsection{Materials and apparatuses}

Dopamine hydrochloride ( $\geq 98 \%$ ), sodium hydroxide ( $\geq 97 \%)$, Lascorbic acid $(\geq 99 \%)$, uric acid ( $\geq 98 \%)$, sulfuric acid $(96 \%)$ were purchased from Sigma-Aldrich (https:// www.sigmaaldrich.com) and used without further purification. All solutions were prepared either in deionized water or in a $0.1 \mathrm{M}$ phosphate-buffered saline (PBS) solution ( $\mathrm{pH}$ 7.4) unless otherwise stated.

Melanin-like nanoparticles were prepared according to the procedure of Ju et al. ${ }^{28}$ In short, $760 \mu \mathrm{L}$ of $1 \mathrm{M} \mathrm{NaOH}$ solution was added to a dopamine hydrochloride solution $\left(2 \mathrm{mg} \mathrm{mL}^{-1}\right)$, prepared by dissolving $180 \mathrm{mg}$ in $90 \mathrm{~mL}$ of deionized water, and the suspension was heated to $50{ }^{\circ} \mathrm{C}$ under continuous stirring for $5 \mathrm{~h}$. As soon as $\mathrm{NaOH}$ was added, the solution color turned to pale yellow and gradually changed to dark brown. The final product (i.e. melanin-like nanoparticles) was isolated by successive centrifugation and deionized water washing.
A Metrohm PGSTAT M204 electrochemical workstation equipped with an FRA32 impedance module controlled by Nova ${ }^{\circledR}$ software was used to record all cyclic and differential pulse voltammetric experiments (CV and DPV) and electrochemical impedance (EIS) measurements. Screen-printed carbon electrodes (SPCEs), incorporating a carbon working electrode, a carbon counter-electrode and a silver pseudoreference electrode, were obtained from Orion Hi-Tech S. L. (Madrid, Spain) and were employed to perform all the electrochemical experiments. SEM micrographs and Energy-Dispersive $\mathrm{X}$-ray Spectroscopy (EDX) analysis were performed using a FEI Quanta FEG250 ESEM scanning electron microscope.

\subsection{Preparation of the modified electrode}

First, the screen-printed carbon electrodes were cleaned and activated using cyclic voltammetry by scanning the potential for 5 cycles in the range $-1.0 \mathrm{~V}$ to $+1.0 \mathrm{~V}$ in $0.5 \mathrm{M} \mathrm{H}_{2} \mathrm{SO}_{4}$ solution. For the electrode preparation, a drop of melanin-like nanoparticles black homogenous suspension $\left(1 \mathrm{mg} \mathrm{mL}^{-1}\right)$, resulting from dispersed melanin-like nanoparticles in deionized water, was deposited on the cleaned electrode surface and was dried for $1 \mathrm{~h}$ at room temperature before use. These devices are designated to be MelNPs/SPCE.

\subsection{Electrochemical measurements}

All assays were run in triplicate by casting $25 \mu \mathrm{L}$ of AA dissolved in a PBS solution on the working electrode. For all the CV and DPV measurements, the scan rate was fixed at $50 \mathrm{mV} \mathrm{s}{ }^{-1}$ and the

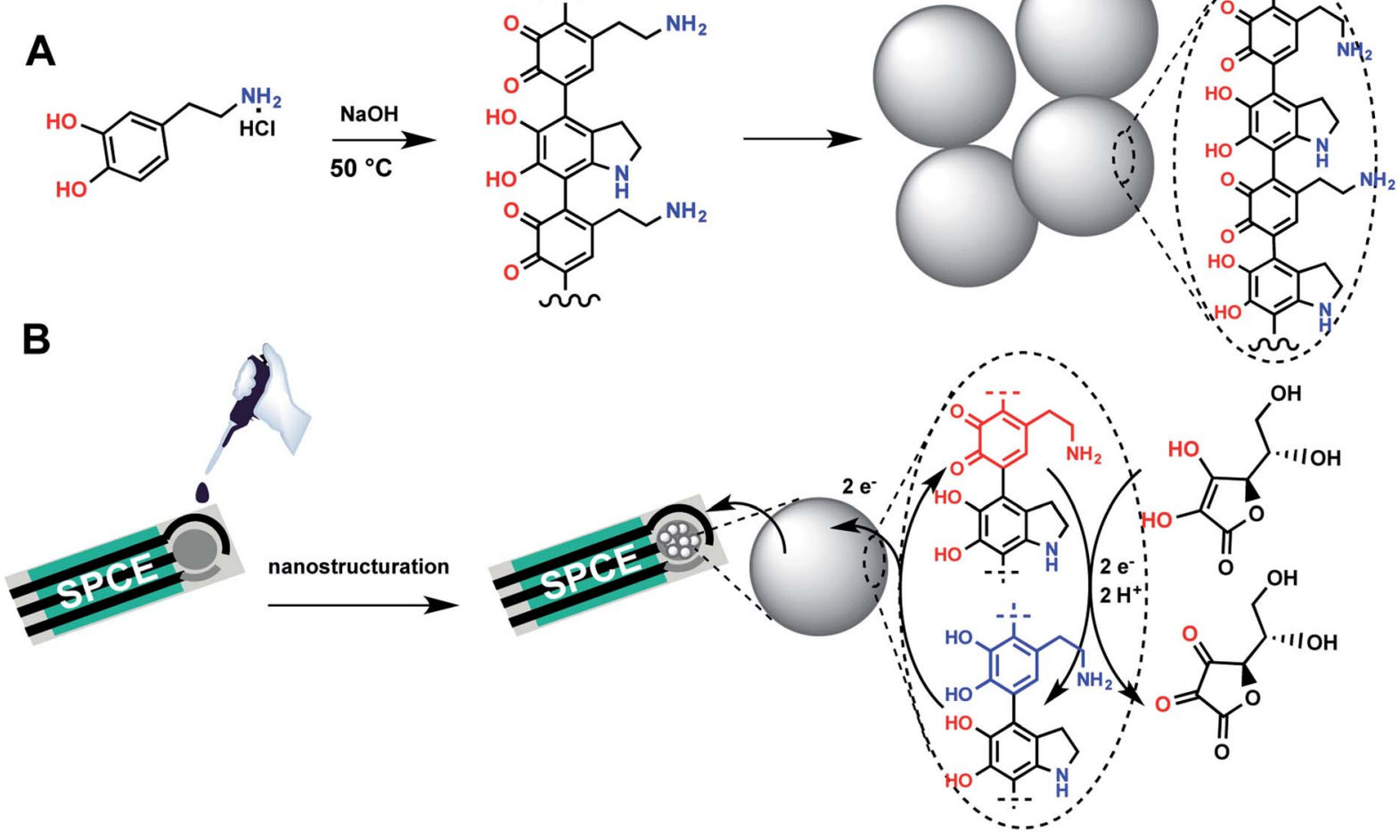

Fig. 1 Principles of the preparation and sensing of ascorbic acid using a melanin-like-modified screen-printed carbon electrode. 
potential range was limited to $[-0.6 \mathrm{~V}, 1.0 \mathrm{~V}]$ prior and after successive additions of $2 \mu \mathrm{L}$ from stock solutions to achieve 0.1 , $0.2,1,2,3,5,10,15,20,50,100,200,300$ or $500 \mathrm{ng} \mathrm{mL} \mathrm{m}^{-1}$ of AA. For the selectivity tests, $25 \mu \mathrm{L}$ (i.e. $50 \mathrm{ng}$ ) of dopamine or uric acid were casted onto the surface of the biosensor, the DPV current was recorded and compared to that obtained for $10 \mathrm{ng}$ of AA. For real samples analysis, human serum, urine and saliva were provided by Prof. Besma Yacoubi-Loueslati (University of Tunis El Manar) and were part of the samples collected for her recent study. ${ }^{29}$ The study was conducted as per Helsinki II declaration and was approved by the Research \& Ethic Committee of Salah Azaïz Institute in Tunis (registration number: ISA/2018/19, granted on 25 June 2017). Informed consent was obtained from all individual participants included in the study. The samples were diluted and no other treatment was carried out.

The impedance spectra were recorded in PBS solution containing $5 \mathrm{mM}$ of $\left[\mathrm{Fe}(\mathrm{CN})_{6}\right]^{4 / 3-}$ at $0.2 \mathrm{~V}$, in a frequency ranging from $100 \mathrm{kHz}$ to $0.1 \mathrm{mHz}$, with an amplitude modulation of $\pm 20 \mathrm{mV}$.

\section{Results and discussion}

\subsection{Characterization and electrochemical properties of Mel- NPs}

The morphology of the Mel-NPs was investigated by scanning electron microscopy and energy dispersive X-ray spectroscopy. As shown in Fig. 2A, the Mel-NPs are highly dispersed with an average size of approximately $200 \mathrm{~nm}$. EDX analysis (Fig. 2B) reveals that they are composed by $c a$. $64 \%$ of carbon, $24 \%$ of oxygen and $12 \%$ of nitrogen, which is approximately the same composition as 2,3-dihydro- $1 H$-indole-5,6-dione, supposedly the molecular unit forming melanin biopolymer. Fig. 2D depicts the stepwise design of the modified Mel-NPs/SPCE using $\left[\mathrm{Fe}(\mathrm{CN})_{6}\right]^{4 / 3-}$ as a redox probe. In fact, a noticeable electron-transfer improvement (i.e. $i_{\mathrm{pc}}$-to- $i_{\mathrm{pa}}$ current ratio and peak-to-peak potential separation) can be observed after the nanostructuration step, which means that Mel-NPs probably served as a shuttle to transport electrons from and to the redox probe towards the electrode surface. Moreover, calculation, using Randles-Sevcik equation, ${ }^{30}$ of the real electrochemical surface area of SPCE before and after modification revealed an enhanced surface area of Mel-NPs/SPCE found to be $1.5 \times 10^{-4} \mathrm{~cm}^{2}$ when compared to unmodified SPCE estimated to be $0.96 \times 10^{-4} \mathrm{~cm}^{2}$, which is in great agreement with the observed improved electrical response. Furthermore, electrochemical impedance spectroscopy, used to probe the changes of electrode surface, shows that the charge-transfer resistance $\left(R_{\mathrm{CT}}\right)$ of the electrode decreased after the deposition of Mel-NPs (Fig. 2E) indicating a better electrical conductivity and hence confirming the surface modification.

Compared to the bare SPCE electrode in a PBS solution, cyclic voltammetry of the Mel-NPs-modified electrode shows a quasi-reversible $\mathrm{CV}$ feature (curve b, Fig. 3A) related to the oxidation and reduction of melanin respectively at $+0.4 \mathrm{~V}$ and $-0.1 \mathrm{~V}$ while the former do not exchange any electrons (curve a, Fig. 3A). The exchanged electrons are due to the oxidation of the melanin hydroquinonoid moiety to their corresponding quinonoid form and their reduction to the former state. Furthermore, the $i_{\mathrm{pc}}$-to- $i_{\mathrm{pa}}$ current ratio close to unity suggests that Mel-NPs keep its reversibility even when adsorbed onto the carbon surface. The oxidation and reduction peak currents increase linearly with scan rates in the range from 10 to $500 \mathrm{mV}$
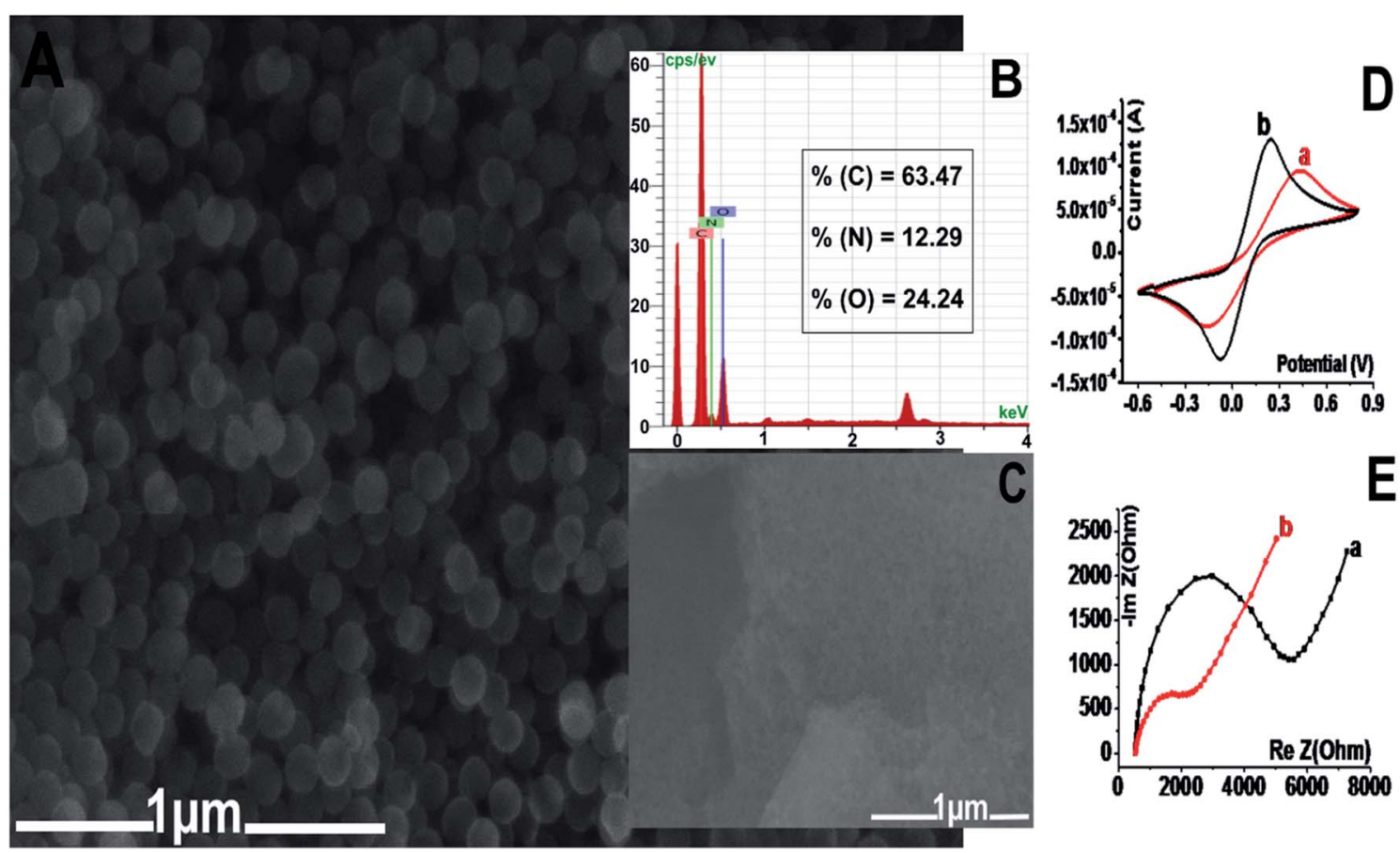

Fig. 2 Characterization of the nanostructured SPCE electrode: (A) SEM image of the modified Mel-NPs/SPCE. (B) EDX analysis of the modified Mel-NPs/SPCE. (C) SEM image of unmodified SPCE. (D) CV and (E) EIS characterization of the SPCE electrode surface: before (a) and after (b) MelNPs deposition. 

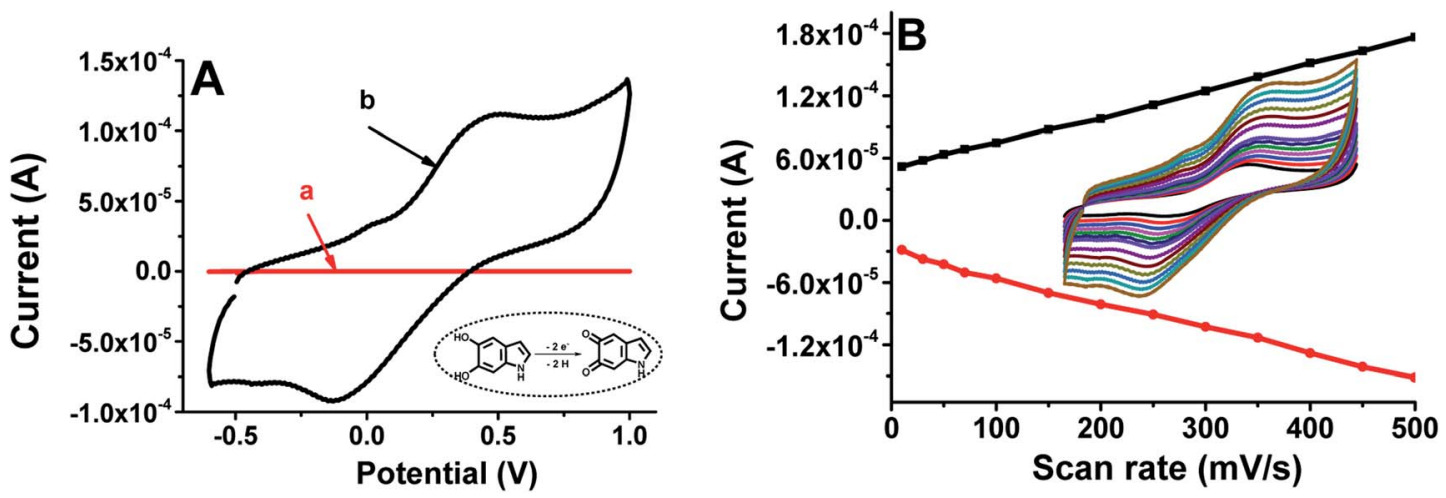

Fig. 3 (A) Cyclic voltammetry curves of the electrode before (a) and after its modification with Mel-NPs (b) in PBS at $50 \mathrm{mV} \mathrm{s}{ }^{-1}$. (B) Plots of anodic and cathodic peak currents vs. scan rates with inset corresponding to CVs of the modified electrode recorded at different scan rates (from inner to outer: $10-500 \mathrm{mV} \mathrm{s}^{-1}$ ).

$\mathrm{s}^{-1}$, suggesting a surface-confined electron transfer process and thus confirming the immobilization of the Mel-NPs on the electrode surface (Fig. 3B). So, we sought to take advantage of the electrochemical features of these Mel-NPs to detect ascorbic acid without using an external redox mediator. In our case, the Mel-NPs will play at once the role of a nanometeric platform modifier and a redox transducer with which we can monitor any surface modification.
3.2. Electrocatalytic activity of Mel-NPs in the presence of ascorbic acid

The electrochemical properties of the bare screen-printed electrode and Mel-NPs/SPCE were examined by cyclic voltammetry in a $0.1 \mathrm{M}$ phosphate-buffered solution PBS containing 1,5 and $10 \mathrm{mM}$ of ascorbic acid at a scan rate of $50 \mathrm{mV} \mathrm{s}^{-1}$. Upon the addition of AA, the shape of the cyclic
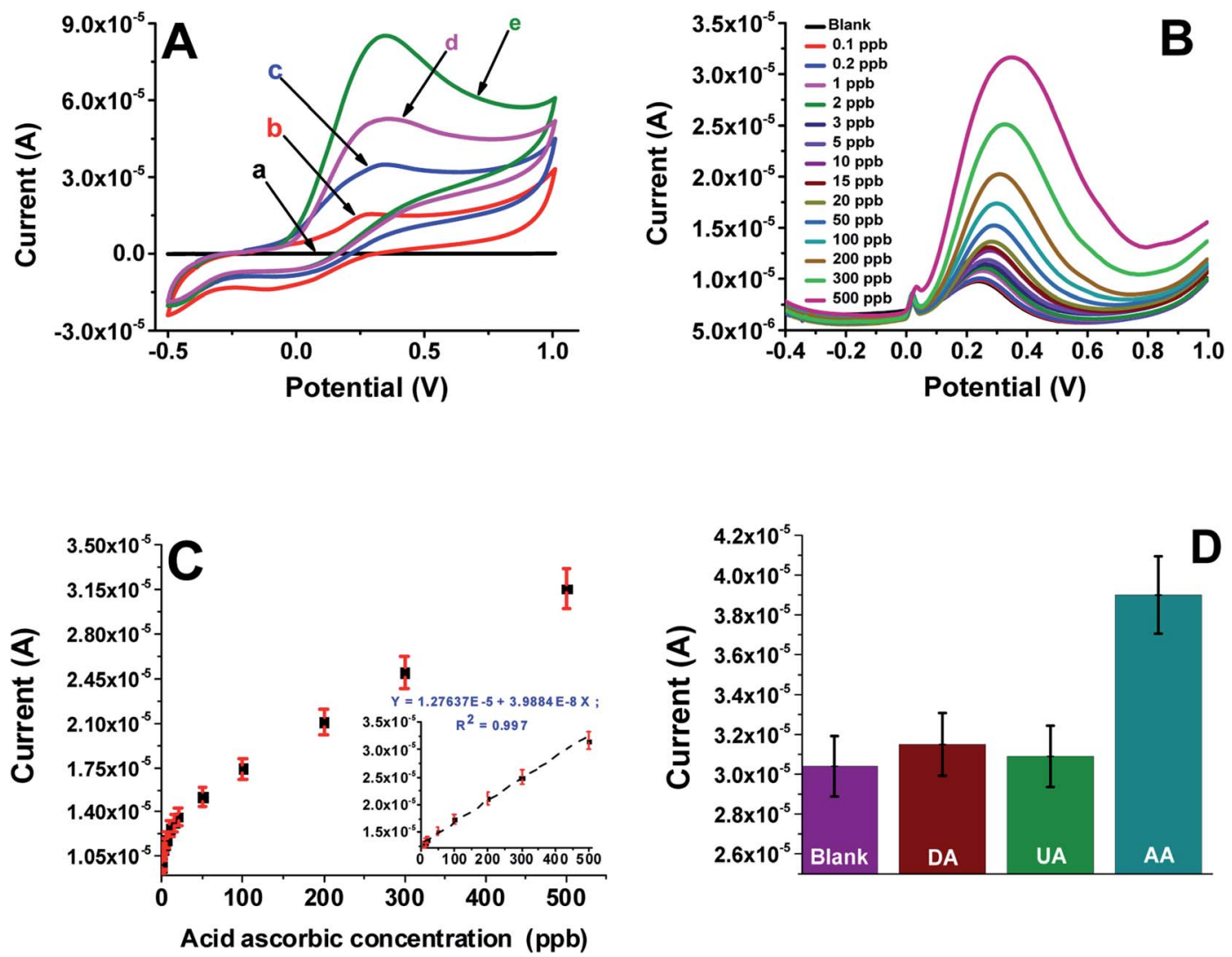

Fig. 4 (A) Cyclic voltammograms of the SPCE (a) and Mel-NPs/SPCE (b) in PBS (0.1 M, pH=7.4) in the absence of ascorbic acid, in PBS containing $1 \mathrm{mM}$ (c), $5 \mathrm{mM}$ (d) and $10 \mathrm{mM}$ (e) ascorbic acid. Scan rate: $50 \mathrm{mV} \mathrm{s}^{-1}$. (B) DPV voltammograms obtained from different concentrations of ascorbic acid in PBS (0.1 M, pH = 7.4). (C) Calibration curve for ascorbic acid detection (the inset shows the linear concentration range). (D) DPV response of the Mel-NPs/SPCE to $10 \mathrm{ppb}$ ascorbic acid and $50 \mathrm{ppb}$ of uric acid and dopamine in PBS $(0.1 \mathrm{M}, \mathrm{pH}=7.4)$. 
voltammograms of the Mel-NPs/SPCE electrode changes significantly to show only a large anodic peak at $c a .+0.30 \mathrm{~V}$ (Fig. 4A) meanwhile, the cathodic peak disappears completely. Increasing the ascorbic acid concentration leads to the anodic current increase. This behavior can be explained by the electrocatalytic reactions that can be schematized using the following equations:

$$
\begin{gathered}
\text { AA } \rightarrow \text { dehydro-AA }+2 \mathrm{H}^{+}+2 \mathrm{e}^{-} \\
\text {Mel-NPs }+2 \mathrm{H}^{+}+2 \mathrm{e}^{-} \rightarrow \mathrm{H}_{2} \text {-Mel-NPs } \\
\mathrm{H}_{2} \text {-Mel-NPs (electrode) } \rightarrow \text { Mel-NPs (electrode) }+2 \mathrm{H}^{+}+2 \mathrm{e}^{-}
\end{gathered}
$$

\subsection{Sensor performances}

The differential pulse voltammetry shows that oxidation current increased steadily with the increase of ascorbic acid concentration. Plotting the current variation versus AA concentration gives a straight with a wide dynamic window ranging from 5 to $500 \mathrm{ppb}$, with a slope of $0.38 \mathrm{nA} \mathrm{ppb}^{-1}$ and $r>0.997$ indicating, respectively, a high sensitivity of the modified electrode and an excellent repeatability of the measurements after three runs. The limits of detection (LoD) and quantification (LoQ) were calculated using the formulae $\operatorname{LoD}=\left(3.3 \times s_{\mathrm{b}}\right) / S$ and $\operatorname{LOQ}=(10$ $\left.\times s_{\mathrm{b}}\right) / S$, respectively, where $s_{\mathrm{b}}$ represents the standard deviation of the blank signal and $S$ represents the sensitivity of the calibration curve. These limits were estimated to be $c a .0 .07 \mathrm{ppb}$ and ca. $0.23 \mathrm{ppb}$, respectively.

Comparison of the sensor performances in terms of limit of detection and linear range with previously reported sensors for AA were summarized in Table 1. As shown, the developed electrode exhibited lower detection limit than that of carbon-supported $\mathrm{NiCoO}_{2}$ nanoparticles, ${ }^{17}$ branch-trunk $\mathrm{Ag}$ hierarchical nanostructure, ${ }^{18} \mathrm{NiFe}_{2} \mathrm{O}_{4}$ nanoparticles, ${ }^{19}$ $\mathrm{MoS}_{2}$ nanosheets, ${ }^{20}$ CuS/Prussian blue core-shell nanohybrid, ${ }^{21}$ and 4 -aminobenzoic acid functionalized herringbone carbon nanotubes ${ }^{22}$ modified electrodes. Whereas, the obtained linear range was smaller than that obtained for others systems. Even known that the AA concentration levels for healthy people in serum are between 30 and $90 \mu \mathrm{M},{ }^{22}$ that the average value of the AA excreted by urine has been established in $132 \mu \mathrm{M}$ (based on a $1.5 \mathrm{~L}$ total urine volume in 24 hours), ${ }^{22}$ and that salivary vitamin $\mathrm{C}$ concentration shown in nonsmokers is in the range of $0.2-19 \mu \mathrm{M}$ with a median of
$0.6 \mu \mathrm{M},{ }^{31}$ determination of $\mathrm{AA}$ in real human serum, urine and saliva samples will be achieved in scarcely diluted samples.

\subsection{Interferences and stability of the modified electrode}

The effect of naturally occurring substances such as dopamine and uric acid that can interfere with the Mel-NPs/SPCE electrode performances in the determination of ascorbic acid in complex media such as human body fluids was evaluated using cyclic voltammetry. The response of the Mel-NPs/SPCE to successive additions of $10 \mathrm{ppb}$ ascorbic acid and five-folds (50 $\mathrm{ppb}$ ) of uric acid or dopamine are displayed in the histogram of the Fig. 4D. Only small increases in the current can be observed with the two substances comparatively to the higher current obtained with $10 \mathrm{ppb}$ of AA. The Mel-NPs/SPCE exhibits an excellent selectivity towards the examined substances especially considering the relative natural abundances in corporal fluid samples such as human serum or saliva.

The repeatability and reproducibility of the developed sensor were evaluated by DPV in a solution containing $10 \mathrm{ppb}$ of AA in a 0.1 M PBS. Using the same modified electrode, the RSD for 5 successive measurements was $5.4 \%$, revealing a good repeatability. Moreover, a RSD of ca. $4.3 \%$, calculated from the responses of 5 independently prepared Mel-NPs/SPCE, is a clear evidence of an acceptable reproducibility. The long-term stability of the Mel-NPs/SPCE electrode was assessed by storing the electrode at RT for 2 weeks in PBS (0.1 M, pH 7.4) and checking its performance daily with the same concentration of AA. The sensor retained $92.7 \%$ of its initial response, indicating good long-term stability.

\subsection{Detection of ascorbic acid in human serum, urine and saliva}

The feasibility of the developed ascorbic acid sensor for practical application was evaluated by detecting the AA concentration in human blood serum, urine and saliva. Standard addition method was adopted according to previous reports. ${ }^{32}$ Different concentrations of AA standard solutions were added to diluted samples of serum $(\times 1000)$, urine $(\times 1000)$ and saliva $(\times 10)$ in order to meet the dynamic range of the sensor. As listed in Table 2 , the recovery values were found to be respectively in the range of $101.3-102.1 \%, 103.7-100.8 \%$ and $107.0-104.8 \%$ with standard derivations of less than $3.4 \%, 3.2 \%$ and $4.1 \%$ in human

\begin{tabular}{|c|c|c|c|}
\hline Electrode materials & Linear range $(\mathrm{M})$ & $\begin{array}{l}\text { Detection } \\
\text { limit (nM) }\end{array}$ & Reference \\
\hline $\mathrm{NiCoO}_{2} / \mathrm{C}$ modified GCE & $10 \times 10^{-6}$ to $2.63 \times 10^{-3}$ & 500.0 & 17 \\
\hline $\mathrm{NiFe}_{2} \mathrm{O}_{4} / \mathrm{SPGE}$ & $5.0 \times 10^{-7}$ to $1.0 \times 10^{-4}$ & 10000.0 & 19 \\
\hline $\mathrm{MoS}_{2}-\mathrm{OCu} / \mathrm{GCE}$ & $0.015 \times 10^{-3}$ to $11.75 \times 10^{-3}$ & 22.2 & 20 \\
\hline CuS@PB/GCE & $5 \times 10^{-6}$ to $3875 \times 10^{-6}$ & 240.0 & 21 \\
\hline hCNTs-4ABA/Au-IDA electrode & 0 to $600 \times 10^{-6}$ & 15000.0 & 22 \\
\hline
\end{tabular}
serum, urine and saliva respectively, indicating the great

Table 1 Comparison of analytical performances of the as-developed Mel-NPs/SPCE sensor with other sensors reported in literature 
Table 2 Detection of AA in real samples of human blood serum, urine and saliva

\begin{tabular}{|c|c|c|c|c|}
\hline Samples & Detected $P$ & Added $Q$ & Detected after addition $R$ & $\begin{array}{l}\% \text { recovery }= \\
100 \times(R-P) / Q( \pm \text { standard deviation })\end{array}$ \\
\hline Serum & 12.45 & 20 & 32.70 & $101.30 \pm 2.80$ \\
\hline \multirow[t]{2}{*}{ Urine } & 17.50 & 20 & 38.24 & $103.70 \pm 3.20$ \\
\hline & 17.50 & 40 & 57.83 & $100.83 \pm 2.60$ \\
\hline Saliva & 140.30 & 20 & 161.7 & $107.00 \pm 4.10$ \\
\hline
\end{tabular}

potential of the Mel-NPs/SPCE electrode for the practical and reliable determination of $\mathrm{AA}$ in real samples. Moreover, the recovery values obtained in all samples were in agreement with those obtained with hCNTs-4ABA/Au-IDA modified electrodes. ${ }^{22}$

\section{Conclusion}

A melanin-like nanoparticles-modified screen-printed carbon electrode was prepared by a simple drop casting procedure and employed to construct an ascorbic acid sensor. Mel-NPs play at once the role of a nanometeric platform modifier and a redox transducer that shows high electrochemical performances for the electrocatalytic oxidation of AA. The developed sensor was successfully employed to detect AA in human body fluids with satisfactory results, revealing its promising practical applicability. Particularly, the prepared electrode may have promising potential applications for AA detection in clinical and biochemical analysis.

\section{Conflicts of interest}

On behalf of all authors, the corresponding authors state that there is no conflict of interest.

\section{Acknowledgements}

The authors acknowledge the help of Prof. Besma YacoubiLoueslati for providing the biological samples collected from consent informed patients and thank PRF NanoFastResponse (ref. PRF2017-D4P1) for financial support and the University of Tunis El Manar for the mobility grants awarded to W. Argoubi and A. Rabti.

\section{References}

1 K. Pallauf, J. Bendall, C. Scheiermann, K. Watschinger, J. Hoffmann, T. Roeder and G. Rimbach, Vitamin C and lifespan in model organisms, Food Chem. Toxicol., 2013, 58, 255-263.

2 G. Maret and F. Jan, Vitamins C and E: beneficial effects from a mechanistic perspective, Free Radical Biol. Med., 2011, 51, 1000-1013.

3 L. Yu, J. Zhao, S. Tricard, Q. Wang and J. Fang, Efficient detection of ascorbic acid utilizing molybdenum
oxide@Prussian Blue/graphite felt composite electrodes, Electrochim. Acta, 2019, 322, 134712.

4 S. Yilmaz, M. Sadikoglu, S. Yagmur and G. Askin, Determination of Ascorbic Acid in Tablet Dosage Forms and Some Fruit Juices by DPV, Int. J. Electrochem. Sci., 2008, 3, 1534-1542.

5 A. R. Mayers and C. G. Taylor, Determination of ascorbic acid in multivitamin tablets by thermometric titrimetry with cerium (IV), Analyst, 1987, 112, 507-509.

6 T. Pérez-Ruiz, C. Martínez-Lozano, A. Sanz and E. Bravo, Simultaneous determination of doxorubicin, daunorubicin, and idarubicin by capillary electrophoresis with laserinduced fluorescence detection, Electrophoresis, 2001, 22, 134-138.

7 X. Wu, Y. Diao, C. Sun, J. Yang, Y. Wang and S. Sun, Fluorimetric determination of ascorbic acid with $o$ phenylenediamine, Talanta, 2003, 59, 95-99.

8 T. Paixão and M. Bertotti, FIA determination of ascorbic acid at low potential using a ruthenium oxide hexacyanoferrate modified carbon electrode, J. Pharm. Biomed. Anal., 2008, 46, 528-533.

9 T. Maki, N. Soh, K. Nakano and T. Imato, Flow injection fluorometric determination of ascorbic acid using perylenebisimide-linked nitroxide, Talanta, 2011, 85, 17301733.

10 P. Barrales, M. Fernández de Córdova and A. M. Díaz, Indirect determination of ascorbic acid by solid-phase spectrophotometry, Anal. Chim. Acta, 1998, 360, 143-152.

11 C. Garnero and M. Longhi, Development of HPLC and UV spectrophotometric methods for the determination of ascorbic acid using hydroxypropyl- $\beta$-cyclodextrin and triethanolamine as photostabilizing agents, Anal. Chim. Acta, 2010, 659, 159-166.

12 M. Noroozifar and M. Khorasani-Motlagh, Solid-phase iodine as an oxidant in flow injection analysis: determination of ascorbic acid in pharmaceuticals and foods by background correction, Talanta, 2003, 61, 173-179.

$13 \mathrm{H}$. Iwase and I. Ono, Determination of Ascorbic Acid in Soups by High-Performance Liquid Chromatography with Electrochemical Detection, J. Agric. Food Chem., 1997, 45, 4664-4667.

14 O. Heudi, T. Kilinç, P. Fontannaz and E. Marley, Determination of vitamin B12 in food products and in premixes by reversed-phase high performance liquid 
chromatography and immunoaffinity extraction, $J$. Chromatogr. A, 2006, 1101, 63-68.

15 A. M. Pisoschi, A. Pop, A. I. Serban and C. Fafaneata, Electrochemical methods for ascorbic acid determination, Electrochim. Acta, 2014, 121, 443-460.

16 Y. P. Dong, L. Huang, J. Zhang, X. F. Chu and Q. F. Zhang, Electro-oxidation of ascorbic acid at bismuth sulfide nanorod modified glassy carbon electrode, Electrochim. Acta, 2012, 74, 189-193.

17 X. Zhang, S. Yu, W. He, H. Uyama, Q. Xie, L. Zhang and F. Yang, Electrochemical sensor based on carbonsupported $\mathrm{NiCoO}_{2}$ nanoparticles for selective detection of ascorbic acid, Biosens. Bioelectron., 2014, 55, 446-451.

18 Y. Zhang, Z. Cai, M. Chen, M. Zhang, P. Liu and F. Cheng, A novel electrochemical ascorbic acid sensor based on branchtrunk Ag hierarchical nanostructures, J. Electroanal. Chem., 2018, 818, 250-256.

19 S. Jahani, Evaluation of the Usefulness of an Electrochemical Sensor in Detecting Ascorbic Acid using a Graphite Screenprinted Electrode Modified with $\mathrm{NiFe}_{2} \mathrm{O}_{4}$ Nanoparticles, Anal. Bioanal. Electrochem., 2018, 10(6), 739-750.

20 D. Li, X. Liu, R. Yi, J. Zhang, Z. Su and G. Wei, Electrochemical sensor based on novel two-dimensional nanohybrids: $\mathrm{MoS}_{2}$ nanosheets conjugated with organic copper nanowires for simultaneous detection of hydrogen peroxide and ascorbic acid, Inorg. Chem. Front., 2018, 5, 112-119.

21 L. Li, P. Zhang, Z. Li, D. Li, B. Han, L. Tu, B. Li, Y. Wang, L. Ren, P. Yang, S. Ke, S. Ye and W. Shi, CuS/Prussian blue core-shell nanohybrid as an electrochemical sensor for ascorbic acid detection, Nanotechnology, 2019, 30, 325501.

22 A. Abellán-Llobregat, C. González-Gaitán, L. Vidal, A. Canals and E. Morallón, Portable electrochemical sensor based on 4-aminobenzoic acid functionalized herringbone carbon nanotubes for the determination of ascorbic and uric acid in human fluids, Biosens. Bioelectron., 2018, 109, 123-131.

23 Y. Liu, K. Ai, J. Liu, M. Deng, H. Yangyang and L. Lehui, Dopamine-Melanin Colloidal Nanospheres: An Efficient
Near-Infrared Photothermal Therapeutic Agent for In Vivo Cancer Therapy, Adv. Mater., 2013, 25, 1353-1359.

24 C. Wang, J. Du, H. Wang, C. Zou, F. Jiang, P. Yang and Y. Du, A facile electrochemical sensor based on reduced graphene oxide and Au nanoplates modified glassy carbon electrode for simultaneous detection of ascorbic acid, dopamine and uric acid, Sens. Actuators, B, 2014, 204, 302-309.

25 D. Nosanchuk and A. Casadevall, Impact of Melanin on Microbial Virulence and Clinical Resistance to Antimicrobial Compounds, Antimicrob. Agents Chemother., 2006, 50, 3519-3528.

26 Y. J. Kim, S. Chun, F. Whitacre and J. Bettinger, Biologically derived melanin electrodes in aqueous sodium-ion energy storage devices, Proc. Natl. Acad. Sci. U. S. A., 2013, 110, 20912-20917.

27 P. Meredith, C. Bettinger, M. Irimia-Vladu, A. Mostert and P. Schwenn, Electronic and optoelectronic materials and devices inspired by nature, Rep. Prog. Phys., 2013, 76, 36.

28 K. Y. Ju, Y. Lee, S. Lee, S. Park and J. Lee, Bioinspired Polymerization of Dopamine to Generate Melanin-Like Nanoparticles Having an Excellent Free-Radical-Scavenging Property, Biomacromolecules, 2011, 12, 625-632.

29 M. Hadj-Ahmed, R. M. Ghali, H. Bouaziz, A. Habel, M. Stayoussef, M. Ayedi, M. Hachiche, K. Rahal, B. Yacoubi-Loueslati and W. Y. Almawi, Transforming growth factor beta 1 polymorphisms and haplotypes associated with breast cancer susceptibility: a case-control study in Tunisian women, Tumor Biol., 2019, 41(8), 1-9.

30 N. P. Shetti, D. S. Nayak, S. J. Malode and R. M. Kulkarni, An electrochemical sensor for clozapine at ruthenium doped $\mathrm{TiO}_{2}$ nanoparticles modified electrode, Sens. Actuators, B, 2017, 247, 858-867.

31 S. J. Padayatty and M. Levine, Vitamin C physiology: the known and the unknown and Goldilocks, Oral Dis., 2016, 22(6), 463-493.

32 W. Sun, Y. Z. Li, Y. Y. Duan and K. Jiao, Direct electrocatalytic oxidation of adenine and guanine on carbon ionic liquid electrode and the simultaneous determination, Biosens. Bioelectron., 2008, 24, 988-993. 